\title{
Whether China's Crude Oil Futures Price Has Become the International Benchmark
}

\author{
Hongzhi TIAN ${ }^{1}$, Hui LI ${ }^{1, *}$ and Feng YAO ${ }^{2}$ \\ 1 Northwest University, Xi'an, China; nwuthz@nwu.edu.cn; Lihui@163.com \\ 2 Kagawa University, Takamatsu, Japan; yao@ec.kagawa-u.ac.jp \\ * Correspondence: Lihui@163.com
}

\begin{abstract}
This paper gives the necessary conditions for judging whether a bulk commodity futures price can become an international benchmark index through the theory of one-way effect causal measure, that is, the conductivity and independence of futures price. Taking Shanghai crude oil futures price as an example, the study finds that: first, the RMB and US dollar prices of SC1809 have a strong influence on WTI and Brent oil prices in a short period and a lasting influence on Brent oil prices in a long period. Second, in terms of the impact on WTI and Brent, the impact of SC1809 RMB price is about 20 times that of US dollar price, which shows that US dollar exchange rate factor absorbs its influence to a large extent, and it is also an important support to maintain the two as benchmark indicators of world oil price. Third, although SC1809 RMB and US dollar prices are not affected by WTI and Brent oil prices in the short term, it only shows a certain degree of isolation. Fourthly, the lack of conductivity of WTI and Brent oil prices indicates that their status as benchmark indicators of world oil prices has been shaken.
\end{abstract}

Keyword: crude oil future prices; conductivity; independence

JEL Classification: G13; C32; C52

\section{Introduction}

As one of the most important primary energy sources, crude oil plays an important role in social and economic development. The fluctuation of crude oil price in recent years has brought great influence to economic growth, and maintaining the stability of crude oil price is an important topic in the sustainable development of mankind. As the world's second largest economy and the largest importer of crude oil, China is more concerned about the volatility of international oil prices. The Shanghai International Energy Exchange, established on March 26, 2018, provides new price reference information for world crude oil futures. What is the impact of the establishment of the futures market on the development of the world crude oil market? Does China, which previously had no international pricing power for crude oil, mean it has the ability to price it? Therefore, this paper analyses a series of market impact problems caused by the generation of Shanghai crude oil futures price, and uses the one-way effect causal measures method to focus on the futures price generated by a futures market as a necessary condition for a commodity international benchmark index.

As the king of bulk commodities, crude oil has undergone many changes in its price evolution path with the great changes of international political and economic situation in recent years. Taking the period from Jan. 7, 2000 to Nov. 11, 2011 as the sample interval, Ji and Fan (2016) used graph theory to study the evolution of world crude oil market and the pricing ability of major oil producing and oil consuming countries. By constructing the minimum spanning tree of world crude oil market, the integration development direction of world crude oil market before 2011 was verified. The paper found that the world crude oil market has the characteristics of coexistence of geographical structure and organizational structure, and that the relationship 
between South America, North America and Africa is relatively stable. The crude oil markets of the United States, Angola and Saudi Arabia are at the core, while the countries of East and Southeast Asia are at the edge of the market. Globalization of the world crude oil market is becoming more stable, but due to the uncertainty of future world politics, its systemic risk may increase. As expected, after 2011, although the international crude oil futures market traditionally uses WTI(West Texas Intermediate) oil price on the New York Mercantile Exchange and Brent oil price on the London Intercontinental Exchange as the world benchmark index, with a series of changes in the international crude oil market, the crude oil price system has also undergone the following new changes:

After 2011, the international benchmark status of Brent and WTI crude oil futures prices has been shaken to varying degrees. In view of the unidirectional and semi-closed shipping flow in the U.S. domestic crude oil market and the characteristics of North American crude oil transportation pipeline network, $\mathrm{Chu}$ and $\mathrm{Xu}$ (2011) considered that the WTI oil price system was no longer suitable as the benchmark index of the world crude oil market. Kilian (2016) pointed out that the North Sea Oilfield is short of liquidity due to production decline. Whether its reserves support Brent crude oil system as a world oil price index in the long run has become a realistic problem facing the world crude oil market. On the basis of these studies, Shi et al.(2018) found that the formation of an internationally influential benchmark price index in a crude oil market requires the following qualitative conditions: a favorable market environment, the geographical location of trading centers, the existence of a variety of market players, active futures and spot markets, an effective regulatory system and transparent market information. These factors all affect whether a certain oil price index can develop into a world benchmark oil price.

As a milestone event in the development of international crude oil futures, the successful operation of Shanghai International Energy Exchange (INE) crude oil futures have attracted wide attention of the academic community. Zhang and Ji (2018) used the data from March 26 to July 31, 2018 to quantify the risk spillover relationship between INE and international benchmark oil price, Shanghai Stock Index and RMB exchange rate. The study found that INE is closely related to WTI and Brent information, but relatively weak to stock market and exchange rate market. In addition, this paper also finds that China's crude oil market is the recipient of information. The information of international oil price fluctuation has a significant positive impact on China's crude oil futures market. China's crude oil futures have not yet formed an independent price discovery mechanism. Shi et al. (2018) used the daily dollar price data from March 26 to August 6,2018 , and found that the daily return rate and intraday volatility of INE index were significantly affected by WTI and Brent, while the reverse was not true. The results show that China's crude oil futures market is still largely affected by the other two markets in the early stage of listing. Contrary to the previous conclusions, Zhou (2018) found that, first, INE price is highly correlated with WTI and Brent oil prices, with a correlation coefficient of more than 0.7, and the change of INE price index has a significant positive pull-up effect on the price changes of both. Second, there is a two-way spillover effect between INE and WTI, Brent futures, and the information transmission between markets is good. Third, compared with the price rising, the price correlation between any two markets will increase in the current three major crude oil futures markets while price declining. It reflects that the China's crude oil futures market has been deeply integrated with the varieties of crude oil futures on the New York Mercantile Exchange and the London Intercontinental Exchange. Wu et al. (2018) found that the price of China's crude oil futures still obeys the "one price law" of international commodities through the study of the impact of the listing of China's crude oil futures on the oil price mechanism, the listing of China's crude oil futures will promote the transfer of oil prices in the Asia-Pacific region to China, and ultimately promote the reform of China's domestic oil price mechanism.

In conclusion, although the Shanghai crude oil futures market has been operating steadily and the trading scale has leaped to the third largest in the world, there are opposite views on the relationship between INE and WTI, Brent oil prices. Therefore, the thinking brought to this paper 
by these previous studies is how to judge the relationship between INE and the two more accurately. It further extends to what is the criterion for a country's futures market to have the international pricing power of certain commodities, or what are the necessary conditions for the international benchmark index of commodity prices. In order to answer these questions, this paper uses the theory of one-way effect causal measure to test the relationship between INE and WTI, Brent.

The contribution of this paper is to creatively give the necessary conditions for judging whether the futures price formed by a futures trading market can become an international benchmark index: conductivity and independence of price. Conductivity refers to the fact that the price of a commodity generated in a country's futures market can affect the transaction price of the same commodity in other markets, which is the reason for the change of other futures price indicators in one-way causality. Independence refers to the fact that the commodity futures price can truly reflect the supply and demand situation of the market. It is not affected by the price of other foreign trading markets. It is expressed by one-way causality that the price is not the result of the change of other oil price indicators. The above two conditions are that when there is only one world benchmark index, if there are two or more world benchmark indicators for a commodity, then there should be no conductivity between these benchmark indicators, while maintaining internal relative independence. Among these three indicators, conductivity and independence are both quantitative indicators. They are confirmed by one-way effect causal measure method.

\section{One-way Effect Causal Measure Theory}

In order to measure the long-term equilibrium and strength of causality of non-stationary macroeconomic time series, Hosoya $(1991,1997)$ has accurately described the second-order stationary process of non-deterministic trend and the interdependence of internal changes of non-stationary process. Three definitions of causality measures in frequency domain and time domain are given and successfully constructed. The early theory of one-way effect causal analysis is established. On this basis, Yao and Hosoya (2000) further gave the statistics of oneway effect causal measure, solved the problem of computer mechanism of multivariable oneway effect causal measure, and established a new method of using one-way effect causal measure to analyze dynamic economic system. The most important feature of this method is that it can test the significance of causality of arbitrary size of multi-economic variables under non-stationary and stationary cases.

Suppose $Z_{t}=\left(X_{t}, Y_{t}\right)^{\prime}$ 'represents $\mathrm{K}$-dimensional column vectors of non-stationary time series, and the dimensions of $X_{t}$ and $Y_{t}$ are $k_{1}, k_{2}$ respectively, and $k=k_{1}+k_{2}$. The Error Correction model of non-stationary time series is as follows:

$$
\Delta Y_{t}=\alpha \beta^{\prime} * Y_{t-1}+\sum_{i=1}^{s-1} \Gamma \Delta Y_{t-i}+\mu+\phi X_{t}+\varepsilon_{t}
$$

In equation (1), $\alpha, \beta$ are the full rank matrix of the $k \times r$ order, where $\beta$ is a vector matrix, $\Gamma$ is a coefficient matrix, $\mu$ is a vector of dimensional constants, $X_{t}$ is a column vector of exogenous variables, $\varepsilon_{t}$ is $k$ dimensional white noise column vector with a covariance matrix of $\Sigma$. After estimating the unknown parameters of Equation (1) using the ordinary least squares, the covariance matrix of the random error term of the model is recorded as $\hat{\Sigma}$, and then $\hat{\Sigma}_{i j}(i, j=1,2)$ means $k_{1}, k_{2}$ order block matrix element, The adjoint matrix of $I_{p}-\left(I_{p}+\right.$ $\left.\hat{\alpha} \hat{\beta}^{\prime}\right) e^{-i \lambda}-\sum_{j=1}^{4} \hat{\Gamma}_{j}\left(e^{-i j \lambda}-e^{-i(j+1) \lambda}\right)$ is $\hat{C}\left(e^{-i \lambda}\right)$, a spectrum response function of $\hat{\Lambda}\left(e^{-i \lambda}\right)=$ $\hat{C}\left(e^{-i \lambda}\right) \hat{\Sigma}^{1 / 2}$ based on the following equation (2):

$$
\hat{f}(\lambda)=\frac{1}{2 \pi} \hat{\Lambda}\left(e^{-i \lambda}\right) \hat{\Lambda}\left(e^{-i \lambda}\right)^{\prime}=\left[\begin{array}{ll}
\hat{f}_{11}(\lambda) & \hat{f}_{12}(\lambda) \\
\hat{f}_{21}(\lambda) & \hat{f}_{22}(\lambda)
\end{array}\right]
$$

The frequency-wise measure of one-way effect (FMO) of $y_{1 t}$ to $y_{2 t}$ can be computed. The following formula (3) shows: 


$$
M_{y_{1} \rightarrow y_{2}}(\lambda \mid \hat{\theta}, \hat{\varphi})=\log \left[\widetilde{\operatorname{det} f}_{11}(\lambda) / \operatorname{det}\left|\begin{array}{l}
\tilde{f}_{11}(\lambda) \\
-\tilde{f}_{12}(\lambda) \tilde{f}_{22}^{-1}(\lambda) \tilde{f}_{21}(\lambda)
\end{array}\right|\right]
$$

where $\tilde{f}_{11}(\lambda)=\hat{f}_{11}(\lambda), \quad \tilde{f}_{21}(\lambda)=\left\{-\hat{\Sigma}_{21} \hat{\Sigma}_{11}^{-1}, I_{k_{2}}\right\} \Lambda(0) \Lambda\left(e^{-i \lambda}\right)^{-1} \hat{f}_{.1}(\lambda), \hat{f}_{1}(\lambda)$ is the initial k column of $\hat{f}(\lambda), \quad \tilde{f}_{22}(\lambda)=\left\{\hat{\Sigma}_{22}-\hat{\Sigma}_{21} \hat{\Sigma}_{11}^{-1} \widehat{\Sigma}_{12}\right\} / 2 \pi$. If the parameter matrix of Equation (1) is rearranged, $\hat{\varphi}$ is $n_{\varphi}=k \times(r+k \times(s-1))+k \times(k+1) / 2$ vector of dimensional, if $\hat{\varphi}=$ $\operatorname{vec}\left(\operatorname{vec}(\hat{\alpha}, \hat{\Gamma})^{\prime}, v(\widehat{\Sigma})\right), \hat{\theta}=\operatorname{vec} \hat{\beta}^{\prime}$, and then, a nonstationary time series the overall measure of one-way effect $(\mathrm{OMO})$ of a pair $y_{1 t}$ to $y_{2 t}$ is defined as:

$$
M(\hat{\theta}, \hat{\varphi})=\frac{1}{\pi} \int_{0}^{\pi} \widehat{M}_{y_{1} \rightarrow y_{2}}(\lambda \mid \hat{\theta}, \hat{\varphi}) d \lambda
$$

OMO can be used to indicate the magnitude of the causal strength. The corresponding Wald statistic is:

$$
\widehat{W}=T\{M(\hat{\theta}, \hat{\varphi})\}^{2} / H(\hat{\theta}, \hat{\varphi})
$$

$H(\hat{\theta}, \hat{\varphi})$ is the variance covariance matrix of $\sqrt{T}\{M(\hat{\theta}, \hat{\varphi})-M(\theta, \varphi)\}$; $\hat{\theta}, \hat{\varphi}$ are the estimated value of $\theta, \varphi$, and the statistic asymptotically obeys the $\chi^{2}$ distribution with a degree of freedom of 1 . The $(1-\alpha) \%$ confidence interval of $M(\hat{\theta}, \hat{\varphi})$ :

$$
\left(M(\hat{\theta}, \hat{\varphi})-\sqrt{(1 / T) H(\hat{\theta}, \hat{\varphi}) \chi_{\alpha}^{2}(1)}, M(\hat{\theta}, \hat{\varphi})+\sqrt{(1 / T) H(\hat{\theta}, \hat{\varphi}) \chi_{\alpha}^{2}(1)}\right.
$$

The detailed deduction process for this model can be referred to Yao and Hosoya (2000).

\section{Empirical Analysis}

In order to investigate whether China's crude oil futures price meets the necessary conditions proposed in this paper, this paper uses one-way effect causal measure theory to calculate the price of INE main contract and the current world crude oil benchmark WTI and Brent respectively, and to examine the one-way effect causal relationship between any two price index systems.

\subsection{Indicator selection and data sources}

China's crude oil futures prices are expressed in terms of INE main contract prices. First, this paper chooses the contract price of SC1809 which is very concerned by all parties in the market. Because one of the pioneering initiatives of Shanghai crude oil futures market is to price and settle accounts in RMB, this paper first uses the RMB price of INE to calculate directly with the US dollar price of WTI and Brent. Second, because crude oil is an important international commodity and international oil prices are priced in US dollars, it is impossible for traders in Shanghai crude oil futures market to ignore the exchange rate of US dollars. In order to consider the influence of these factors, we use the exchange rate of RMB to US dollar to convert the INE RMB price into US dollar price, and then measure the one-way effect causality with WTI and Brent prices under the same currency valuation system.

Because the Shanghai International Energy Exchange Center stipulates that the last trading day of the month before the delivery month shall be the last trading day, and the main contract of SC1809 shall be delivered in September 2018, the sample interval of this paper is determined from March 26, 2018 to August 30, 2018. The variables in the model are defined as follows: $S C 1809 R_{t}$ denotes the daily frequency RMB price of SC1809 main contract in INE market, and $S C 1809 D_{t}$ denotes the daily frequency US dollar price of SC1809 main contract in INE market. $W T I_{t}$ represents the daily price of WTI crude oil futures on the New York Mercantile Exchange in the sample range, while $B R E N T_{t}$ represents the daily price of Brent crude oil futures on the London Intercontinental Exchange, both of which are priced in US dollar.

RMB price data of INE comes from the daily settlement price of Shanghai International Energy Exchange. WTI price of crude oil futures on the New York Mercantile Exchange is 
collected on the official website of the U.S. Energy Information Administration. Brent crude oil futures price on the London Intercontinental Exchange is extracted from the CEIC database. The data of RMB exchange rate against the US dollar are from the mid-price data of the official website of the People's Bank of China.

\subsection{Statistical characteristics and stationarity test of data}

According to the stationarity of time series, one-way effect causal measures have different measurement methods, and the precondition of co-integration among variables is that each sequence has the same differential stationarity process, so we first verify the stationarity of the level values and the first-order difference of the selected sequence. This paper uses ADF (Augmented Dickey-Fuller test) method to test. The results are listed in Table1. It shows that the variables selected in this paper are non-stationary time series under level values, and stable after first-order difference. This shows that all four series of data obey the $I(1)$ process and satisfy the condition of one-way effect causal measure.

Table 1. Stability test of variables.

\begin{tabular}{ccccc}
\hline Variable & $\begin{array}{c}\text { Level value } \\
\text { series }\end{array}$ & Stationarity & $\begin{array}{c}\text { First-order } \\
\text { difference series }\end{array}$ & Stationarity \\
\hline$S C 1809 R_{t}$ & -1.4984 & non- & $-9.1470^{* * *}$ & stable \\
& $(0.5308)$ & stationary & $(0.0000)$ & \\
$S C 1809 D_{t}$ & -1.9743 & non- & $-9.5322^{* * *}$ & stable \\
& $(0.2977)$ & stationary & $(0.0000)$ & stable \\
$W T I_{t}$ & -2.2848 & non- & $-11.1239^{* * *}$ & $(0.0000)$ \\
& $(0.1783)$ & stationary & stable \\
BRENT & -2.4594 & non- & $-11.8295^{* * *}$ & $(0.0000)$ \\
\hline
\end{tabular}

Note: “***” means rejecting the null hypothesis at the $1 \%$ significance level. The values in parentheses below the statistics are the corresponding $p$ values.

\subsection{Testing of cointegration relations among variables}

It is noteworthy that this paper finds that there is no co-integration relationship between WTI and Brent prices. Although their prices reflect the common trend in the world supply and demand environment, the movement process is relatively independent, and both of them have the function of price discovery that reflects the reality of local futures market transactions.

Table 2. Cointegration relationship between variables.

\begin{tabular}{ccccc}
\hline Variable group & \multicolumn{2}{c}{$\begin{array}{c}\text { Characteristic roots and } \\
\text { characteristic vectors }\end{array}$} & $\begin{array}{c}\text { Cointegration } \\
\text { rank }\end{array}$ & Trace statistics \\
\hline & $(0.0809$ & $0.0520)$ & $2-\mathrm{r}$ & \\
$S C 1809 D_{t}$ & 0.9956 & -0.5610 & 1 & $5.6030^{* *}$ \\
$W T I_{t}$ & -0.0941 & 0.8278 & 2 & $14.4590^{*}$ \\
& $(0.0744$ & $0.0335)$ & $2-\mathrm{r}$ & \\
$S C 1809 D_{t}$ & 0.1168 & 0.8331 & 1 & $3.5720^{*}$ \\
$B R E N T_{t}$ & 0.9932 & -0.5531 & 2 & 11.6890 \\
& $(0.0979$ & $0.0316)$ & $2-\mathrm{r}$ & \\
$S C 1809 R_{t}$ & 0.9998 & 0.9681 & 1 & $3.3418^{*}$ \\
$W T I_{t}$ & -0.0198 & -0.2507 & 2 & $14.0568^{*}$ \\
& $(0.0677$ & $0.0260)$ & $2-\mathrm{r}$ & \\
$S C 1809 R_{t}$ & 0.9994 & 0.9923 & 1 & $2.7431^{*}$ \\
\hline
\end{tabular}




\begin{tabular}{clccc}
\hline BRENT & 0.0347 & -0.1237 & 2 & 10.0320 \\
& $(0.0751$ & $0.0308)$ & $2-\mathrm{r}$ & \\
$W T I_{t}$ & 0.0870 & 0.7915 & 1 & 2.6900 \\
$B R E N T_{t}$ & 0.9962 & -0.6112 & 2 & 13.3300 \\
\hline
\end{tabular}

Note: The ${ }^{* *}, * * n$ following the trace statistic indicates the rejection of the null hypothesis at the $10 \%$ and $5 \%$ significance levels, respectively.

\subsection{One-way effect causality test}

Because the two dominant markets of crude oil futures in the world are the New York Mercantile Exchange and the London Intercontinental Exchange, in order to investigate the world position of China's crude oil futures prices, we only need to measure the conductivity and independence between INE, WTI and Brent.

\subsubsection{Conductivity test of Shanghai crude oil futures price}

Table 3 gives the calculation results of INE's one-way full measure and confidence interval for WTI and Brent. The results show that both RMB and US dollar prices, Shanghai crude oil futures have a significant impact on WTI and Brent oil prices, and have conductivity. INE has attracted intense attention from the New York Mercantile Exchange and the London Intercontinental Exchange, and has had a substantial impact on them. The OMO in Table 3 shows that the one-way effect causal intensity of INE RMB price to WTI and Brent are 24.5 times and 21.5 times as much as that of US dollar price, respectively. It can be seen that the use of RMB valuation in Shanghai crude oil futures trading has greatly enhanced the international influence of INE price, increased the world status of the domestic crude oil futures market, and reflected the foresight at the beginning of the construction of the crude oil futures market in Shanghai.

Table 3. One-way effect causality measure of INE to WTI and Brent.

\begin{tabular}{cccc}
\hline $\begin{array}{c}\text { One-way effect } \\
\text { causality }\end{array}$ & OMO & Wald statistic & $\begin{array}{c}\text { 95\% confidence } \\
\text { interval }\end{array}$ \\
\hline$S C 1809 D_{t}$ to $W T I_{t}$ & 0.0874 & $\begin{array}{c}9.5964^{* * *} \\
(0.0020)\end{array}$ & $(0.0321,0.1426)$ \\
$S C 1809 D_{t}$ to $B R E N T_{t}$ & 0.1191 & $\begin{array}{c}12.0989^{* * *} \\
(0.0005)\end{array}$ & $(0.0520,0.1863)$ \\
$S C 1809 R_{t}$ to $W T I_{t}$ & 2.1394 & $\begin{array}{c}7.9175^{* * *} \\
(0.0049)\end{array}$ & $(0.6492,3.6297)$ \\
$S C 1809 R_{t}$ to $B R E N T_{t}$ & 2.5627 & $7.0765^{* * *}$ & $(0.6746,4.4509)$ \\
& & $(0.0078)$ & 1.3307 \\
& & $(0.2487)$ & $(-0.1204,0.4647)$ \\
$B R I_{t}$ to $B R E N T_{t}$ & 0.17218 & 1.7724 & $(-0.0784,0.4107)$ \\
\hline
\end{tabular}

Note: the Wald statistics below are in parentheses for their corresponding $p$ values, and " $* * * *$ denotes the rejection of the null hypothesis without causality at the $1 \%$ significance level, respectively.

It should be emphasized that, following the fact that there is no co-integration relationship between WTI and Brent, Table 3 shows that there is no causal relationship in any direction between WTI and Brent, indicating that they are not conductive to the other side and that they are mutually independent. It should be emphasized that there are two benchmark index systems of WTI and Brent in the world crude oil, which are recognized by the world market. 
Therefore, in this case, there should be no conductivity between them, but an independent index system, which shows the internal independence between the indicators. The results in Table 4 confirm that after decades of baptism in the international crude oil market, both of them have an independent mechanism for generating benchmark crude oil price indicators, which conforms to the existing pattern of the international crude oil market.

Although Table 3 gives the one-way effect causal measure between the three oil price indicators, according to the supply and demand situation of crude oil, there may be complex influence process and mechanism on other crude oil futures markets after the futures price is generated through trader's transaction by purpose of hedge and arbitrage. In order to confirm this problem, we further describe the short, medium and long-term changes of Shanghai crude oil futures price in time. In this paper, the spectrum measures are arranged in Figures 1-4. Different from the time domain analysis method, the spectrum analysis theory regards time series as the superposition of different harmonics, and the product of period and frequency is equal to $2 \pi$. The horizontal axes in the following graphs represent the spectrum domain, and the long-term causal relationship between variables near the origin $0.0 \pi$ corresponds to infinite number of cycles; the short-term causal relationship near $1.0 \pi$ corresponds to two sample cycles, i.e. the unidirectional causal influence intensity of two-day cycles. Because the spectrum domain has internal symmetry $(-\pi, \pi]$, we only draw the cases between $[0, \pi]$.

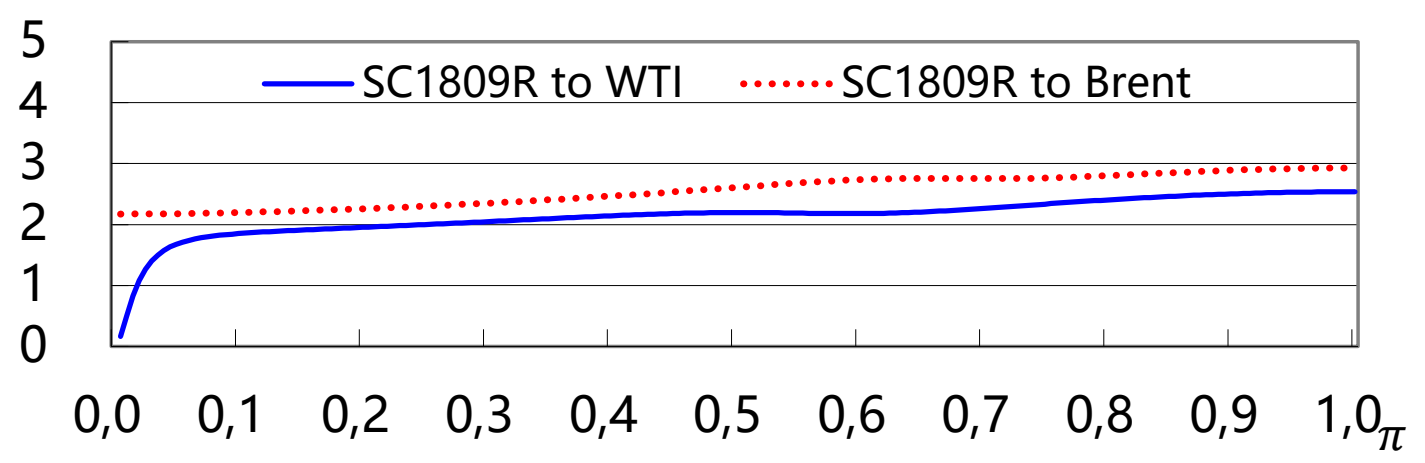

Figure 1. Distribution map of one-way spectrum measurement of SC1809 RMB price to WTI and Brent.

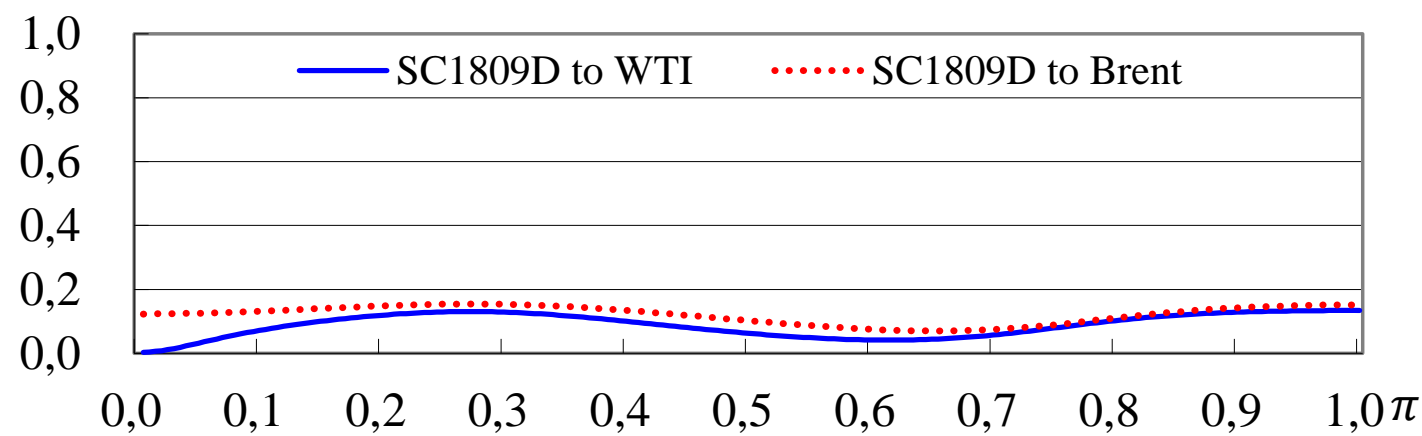

Figure 2. Distribution map of one-way spectrum measurement of SC1809 Dollar price to WTI and Brent.

Although the operation time of Shanghai crude oil futures market is relatively short, it has grown rapidly to the third largest crude oil futures market in the world. Figure 1 shows that the settlement price of RMB reached by SC1809 main contract has a significant unilateral causal relationship with WTI oil price in both short and medium cycles. The impact process started two days after the price of SC1809 RMB was generated. At first, the impact intensity was the strongest, then gradually attenuated, and finally attenuated to 0 . That is to say, WTI is not 
affected by Shanghai crude oil futures market and has long-term independence. The difference between Brent and WTI is that, firstly, Figure 1 shows that the RMB price of SC1809 not only has a significant and robust impact on Brent in short and medium cycles, but also maintains its influence in long cycles, indicating that the one-way effect causal relationship between them is relatively stable; secondly, the influence of RMB settlement price of SC1809 main contract on Brent is stronger than WTI oil price, which indicates that the relationship between Shanghai crude oil futures market and the London Intercontinental Exchange is closer than that of the New York Mercantile Exchange. It can be seen from the above that the one-way effect causal measure can not only measure the change of one-way effect causal in time, but also give the strength of causality.

Because the exchange rate of RMB against US dollar in the same period may be a comprehensive reflection of the world's macroeconomic fluctuations and various economic relations, we also calculated the causal relationship between the price of US dollar SC1809 and WTI and Brent. In the short period of the right spectrum domain in Figure 2, the impact of SC1809 price on WTI is the greatest in 2 days (i.e. 1.0 $\pi$ ), and then there is a significant causal strength decline in the period of $0.625 \pi$, i.e. about 3 days. Then it reaches its maximum in the period of $0.28 \pi$, i.e. 7 days, and finally the long-term effect is completely attenuated to 0 . This shows that one of the main factors that WTI crude oil price is independent of INE impact is the US dollar exchange rate. Before 2018, the United States had been the world's largest importer of crude oil. With the increase of domestic crude oil production in recent years, the United States hoped to control oil prices through the dollar exchange rate factor, which objectively made WTI oil price to a certain extent out of the supply and demand situation of the international crude oil market, but only reflected the supply and demand situation in the domestic market of the United States.

In Figure 2, the trend of Brent in short and middle cycle is basically the same as that of WTI. It is shown again that the main factor of this situation is the dollar exchange rate factor, which weakens the influence of INE on Brent oil price. The biggest difference between Brent and WTI is that the long-term impact of INE on Brent oil prices still exists, even in the US dollar pricing scenario. Considering the geographic factors, the most significant difference between Brent and WTI crude oil futures market is that Brent crude oil futures market is located in London, which is the hub of Eurasian crude oil futures market. Its supply and demand are not only directly affected by European countries, but also by Asian crude oil demand. The strong demand for crude oil in China, India and other Asian emerging market countries in the past decade is directly reflected in the price changes of crude oil futures on the London Intercontinental Exchange. It can be seen that as the largest crude oil futures market in Asia, the trading price of Shanghai International Energy Exchange is closely linked with the crude oil futures market in London even if it includes the factor of dollar exchange rate.

Comparing the influence of INE RMB price and US dollar price on WTI and Brent in Figure 1 and Figure 2, we can find that the causal strength of RMB price system is more than 20 times that of US dollar system. This conclusion shows that the US dollar exchange rate has greatly reduced the market influence of INE. Conversely, the Shanghai International Energy Exchange uses RMB to price, which is very helpful to expand the international influence of China's crude oil futures market.

\subsubsection{Independence test of Shanghai crude oil futures price}

Table 4. One-way effect causality measure of WTI and Brent to INE.

\begin{tabular}{cccc}
\hline $\begin{array}{c}\text { One-way effect } \\
\text { causality }\end{array}$ & OMO & Wald statistic & $\begin{array}{c}\text { 95\% confidence } \\
\text { interval }\end{array}$ \\
\hline$W T I_{t}$ to $S C 1809 D_{t}$ & 0.3015 & $\begin{array}{c}2.4863 \\
(0.1148)\end{array}$ & $(-0.0733,0.6764)$ \\
\hline
\end{tabular}




\begin{tabular}{cccc}
\hline BRENT & to $S C 1809 D_{t}$ & 0.9202 & $(-0.1464,0.4270)$ \\
& 0.1403 & $(0.3374)$ & \\
& & 0.5325 & \\
$W T I_{t}$ to $S C 1809 R_{t}$ & 0.0233 & $(0.4656)$ & $(-0.0392,0.0858)$ \\
& & 0.9441 & $(-0.0001,0.0002)$ \\
BRENT & 0.0001 & $(0.3312)$ & \\
\hline
\end{tabular}

Note: the Wald statistic is given in parentheses below for its corresponding $p$ value.

From Table 4, we can see that the P value corresponding to the Wald statistics of one-way effect causal measure is larger, accept the original hypothesis that neither WTI nor Brent oil price can cause the change of dollar price and RMB price of SC1809 main contract, which indicates that Shanghai crude oil futures price is not affected by the other two. This table shows that although Shanghai crude oil futures have a short listing time, they are not affected by other crude oil futures markets because of their accurate market positioning, reasonable valuation and currency selection, and even withstand the pressure of the fluctuation of US dollar exchange rate. So, according to this conclusion, can we conclude that Shanghai crude oil futures really have independence? Although the one-way effect causal measure of WTI and Brent is not statistically significant for Shanghai crude oil futures price, the one-way effect causal spectrum measure distribution map can give detailed information about the process of their action on Shanghai crude oil futures price and the specific changes in time. Figures 3 and 4 can give us some references.

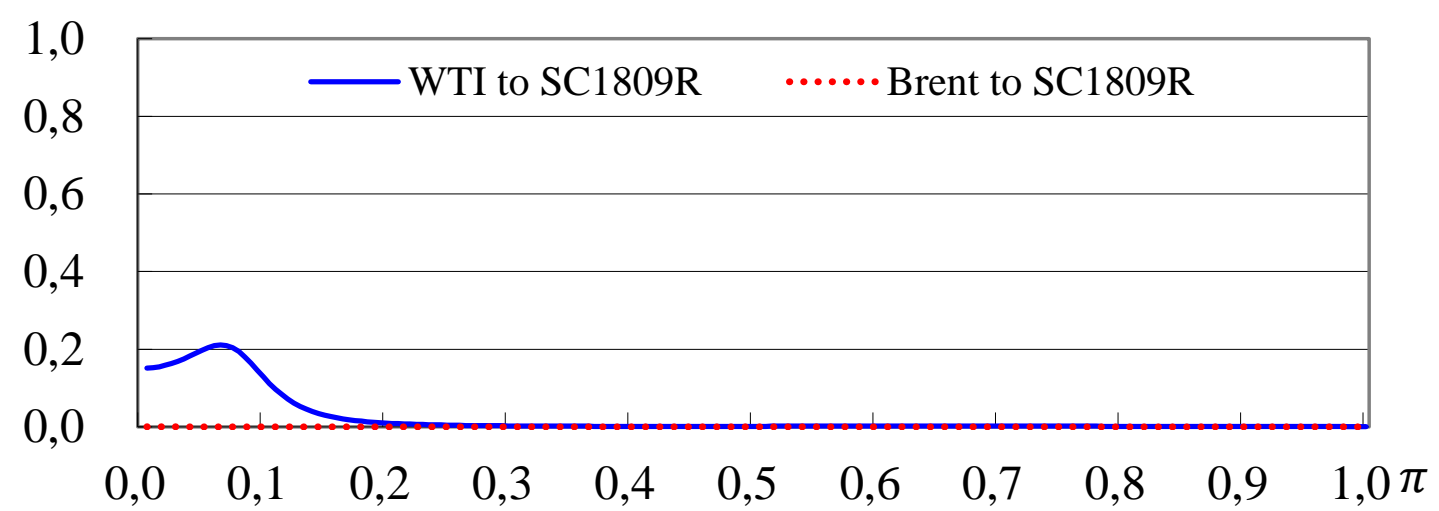

Figure 3. Distribution map of one-way spectrum measurement of SC1809 RMB price to WTI and Brent.

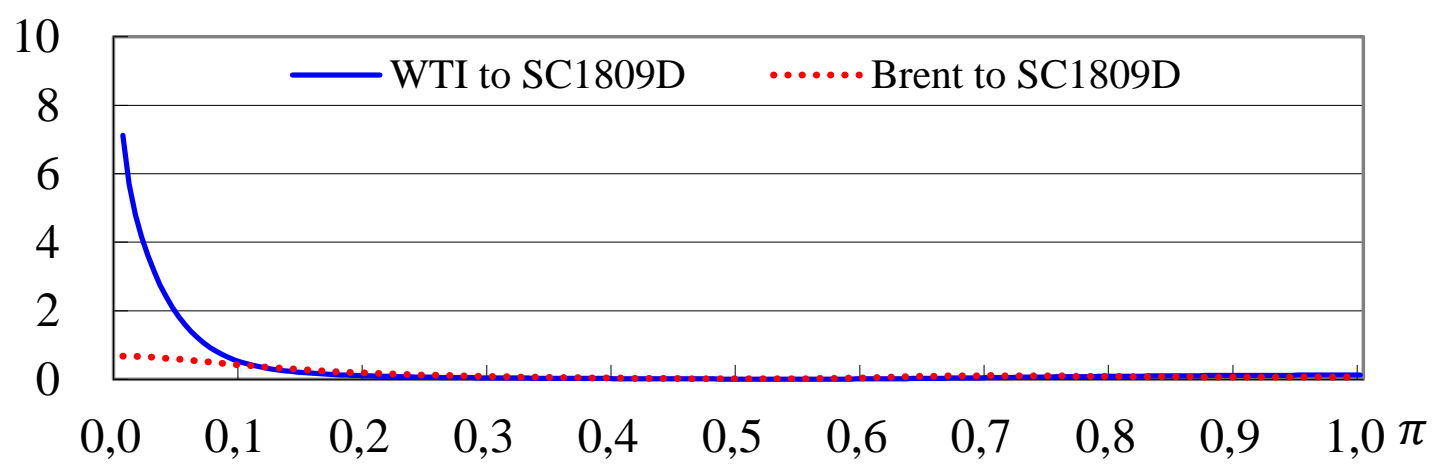

Figure 4. Distribution map of one-way spectrum measurement of SC1809 Dollar price to WTI and Brent. 
Figure 3 shows that WTI and Brent have no significant impact on the price of Shanghai crude oil futures in the short and medium term, and only have a certain degree of influence over the long term. In line with the conclusion in Figure 1, INE does not affect WTI in the long run, but WTI can affect INE. Similarly, consistent with the conclusion in Figure 1, Brent did not have a significant impact on INE in the long run. Compared with Figure 3, Figure 4 shows that WTI and Brent can influence INE only in the long run with the help of dollar exchange rate factors.

From the above analysis, Table 4 calculates that the independence of INE is essentially short-term independence. In the long run, it may still be affected by WTI and Brent to a certain extent, and this effect is significantly strengthened under the US dollar exchange rate factor. Therefore, the short-term independence of Shanghai crude oil futures in the early stage of operation is essentially another kind of situation - isolation. The difference between isolation and independence is that independence means that the information received from other futures markets is not affected even if it receives information from other futures markets for a long time. However, the isolation is that the Shanghai crude oil futures market is at the end of the information, the information received in other futures markets is limited in the short term, and the number of international traders and trading volume are very small, so it has not been able to have a substantial impact on it.

\section{Conclusions}

Under the circumstance that commodity futures price determines spot price, China has made a lot of efforts to gain international pricing power for commodities. China established the Shanghai crude oil futures market in 2018 in order to make a Chinese voice on the international stage. The traditional Granger causality test can only judge whether there is causality between two variables, and it can't capture carefully the change of causality and causality intensity in short and medium periods. Therefore, based on the theory of unidirectional causality measure, this paper analyses the causal direction and intensity of crude oil futures prices between the New York Mercantile Exchange, the London Intercontinental Exchange and the Shanghai International Energy Exchange, which are among the top three trading volumes in the world, in order to identify whether the formation mechanism of China's crude oil futures prices has met the necessary conditions for becoming an international benchmark index. The main conclusions of this paper are as follows: First, in a short period of 1-3 days, the RMB and US dollar prices of SC1809 have a strong influence on WTI and Brent oil price indicators; in the long period, the RMB and US dollar prices of SC1809 both have a permanent impact on the price of the Brent oil, indicating that the INE oil price already has the conductivity. Second, in terms of the impact of INE on WTI and Brent, the impact of its RMB price is about 20 times that of the US dollar which shows that US dollar exchange rate factor absorbs INE's market influence to a large extent, thus maintaining the independence of WTI and Brent oil prices, and is also an important factor for them to remain as benchmark indicators of world oil prices.

\section{References}

Chu Wangtao, Xu Zhaohui. 2011. Causes and influence of the WTI-Brent spread anomaly (in Chinese). International Petroleum Economics: 19(09), 7-14+108. https://doi.org/10.3969/j.issn.10047298.2011.09.002

Hosoya Y. 1997. A limit theory for long-range dependence and statistical inference on related models. The Annals of Statistics: 25(1), 105-137. https://doi.org/10.2307/2242714 
Hosoya Y. 1991. The decomposition and measurement of the interdependency between second order stationary processes. Probability Theory and Related Fields: 88(4), 429-444. https://doi.org/10.1007/bf01192551

Ji Q, Fan Y. 2016. Evolution of the world crude oil market integration: A graph theory analysis. Energy Economics: 53(1), 90-100. https://doi.org/10.1016/j.eneco.2014.12.003

Kilian L. 2016. The impact of the shale oil revolution on US oil and gasoline prices. Review of Environmental Economics and Policy: 10, 185-205. https://doi.org/10.1093/reep/rew001

Shi Xunpeng, Ji Qiang, Zhang Dayong. 2018. Evolution of international crude oil pricing mechanism and its implications to China's crude oil futures(in Chinese). Journal of Environmental Economics: 3, 121134. https://doi.org/10.19511/j.cnki.jee.2018.03.009

Wu Jinwen, Zhang Weiqing, Zhang Rong. 2018. Research on the impact of China's crude oil futures listing on oil price mechanism(in Chinese). China Chief Financial Officer: 10, 46-48. https://doi.org/10.3969/j.issn.1672-576X.2018.10.017

Yao F, Hosoya Y. 2000. Inference on one-way effect and evidence in Japanese macroeconomic data. Journal of Econometrics: 98, 225-255. https://doi.org/10.1016/S0304-4076(99)00084-6

Zhang Dayong, Ji Qiang. 2018. Studies on the dynamic risk spillovers for China's crude oil futures (in Chinese). Chinese Journal of Management Science: 26, 42 - 49. https://doi.org/10.16381/j.cnki.issn1003207x.2018.11.005

Zhou Yinggang. 2018. China's crude oil futures are well linked with the global market (in Chinese). Financial Times, 06-28(008). 